\title{
Capillary Action Around Dental Structures
}

\author{
W. J. O'BRIEN \\ School of Dentistry, University of Michigan, Ann Arbor, Michigan 48104, USA
}

The capillary action of saliva occurs in the crevices around and between teeth and around dental restorations. Marginal leakage and denture retention caused by a thin film of saliva are aspects of capillary phenomena. Liquids in capillaries isolated from a reservoir showed an increase in surface tension and lower vapor pressure. The strength of thin films of human saliva was independent of ambient pressure.

Capillary penetration around dental restorations recently has been studied in connection with marginal leakage and denture retention. The penetration of liquids between plates slightly inclined to each other forms a hyperbola (Fig 1) given by the equation ${ }^{1}$

$$
h X=\frac{\gamma_{L V}\left(\cos \theta_{1}+\cos \theta_{2}\right)}{2 d g \tan \phi / 2},
$$

where $\phi$ is the angle between the plates, $h$ is the rise height at a distance $X$ from the vertex, $g$ is the gravitational constant, $\gamma_{L V}$ is the surface tension of the liquid, $d$ is the liquid density, and $\theta_{1}$ and $\theta_{2}$ are the liquidsolid contact angles. The relation of degree of penetration, crevice distance, and wetting is illustrated in Figure 2. Maximum penetration results when the liquid wets the solid completely and the crevice is narrow.

Another aspect of capiliary phenomena is the adhesion of liquid bridges. This adhesive force has been identified as a contributing factor in denture retention. According to classical theory, the pressure below a concave meniscus is less than that of atmospheric pressure. ${ }^{2}$ Also, a separate component caused by surface tension usually is added to this reduced pressure term. Recently, O'Brien ${ }^{3,4}$ derived the equation for

This Research was supported by USPHS Grant DE03480 from the National Institute of Dental Research, National Institutes of Health, Bethesda, Md.

Received for publication April 28, 1972. the strength of liquid bridges between two spheres on the basis of an increased liquidsurface tension term alone. An experiment to test the effect of atmospheric pressure on the strength of liquid bridges was suggested by this approach.

The difference between isolated capillaries (isocaps) and connected capillary systems is illustrated in Figure 3. Isolated capillaries are formed when liquids are trapped in crevices isolated from a reservoir. As a result of liquid-solid attraction, the vacancy concentration at the liquid-vapor interface is increased, and this causes an increase in intermolecular attraction. This increased attraction manifests itself as an increase in liquid-surface tension and a higher bridge strength.

To minimize the effect of liquid evaporation, the bridge between a spherical lens and a flat plate was chosen, since the strength of these bridges is independent of film thickness for small amounts of liquid. ${ }^{5}$

The equation for the strength of this bridge based on increased surface tension may be derived with reference to Figure 4 . The increased liquid-surface tension, $\gamma_{L}{ }^{*}$, is given by the relation ${ }^{3}$

$$
\gamma^{*}{ }_{L}=\gamma_{L}+\frac{\left(\gamma_{S V}-\gamma_{S L}\right) A_{S L}}{A_{L V}},
$$

where $\left(\gamma_{S V}-\gamma_{S L}\right)$ represents the free energy term on wetting and $A_{S L}$ and $A_{L V}$ are the solid-liquid and liquid-vapor interfacial areas. The value of $\gamma^{*}$ is obtained from equation 1 by use of the following geometrical approximations for low $\psi$ values,

$$
A_{L V}=\pi^{2} s b,
$$

and

$$
A_{S L}=2 \pi s^{2} .
$$

Substitution of $\gamma \cos \theta$ from the Dupre 


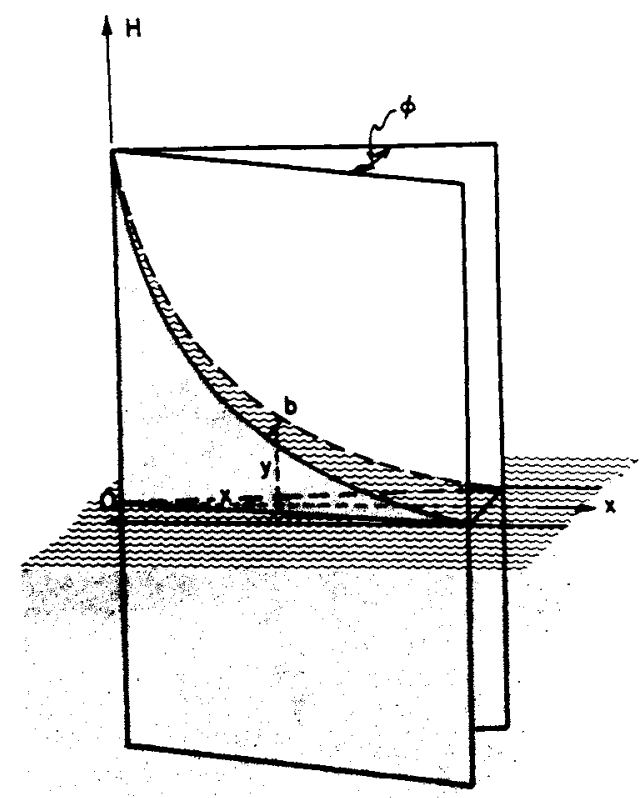

FIG 1.-Capillary rise between plates at an angle.

equation for $\left(\gamma_{S V}-\gamma_{S V}\right)$ gives the following equation

$$
\gamma^{*}=\gamma+\frac{2 s \gamma \cos \theta}{\pi b}
$$

The work necessary to stretch the menis-

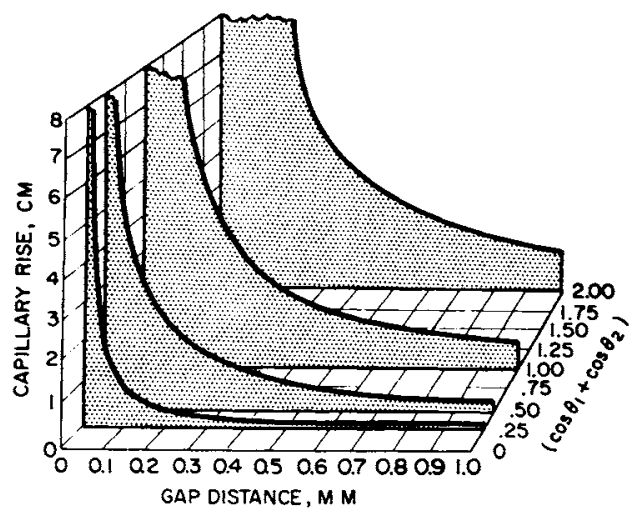

Fig 2.-Capillary rise curves for water between two plates with different wettabilities.

cus film by an amount $\delta b$ is equated to the increase in surface energy of the meniscus

$$
F \delta b=\gamma^{*} \pi^{2} s \delta b,
$$

where $\pi^{2} s \delta b$ is the increase in surface area.

If the value of $\gamma^{*}$ from equation 4 is substituted, the following is obtained

$$
F=\gamma \pi^{2} s+\frac{2 \gamma \cos \theta \pi s^{2}}{b}
$$

At angles of $\psi$ of a few degrees

$$
s^{2}=2 R b
$$

which gives

$$
F=\gamma \pi^{2} s+4 \pi R \gamma \cos \theta
$$

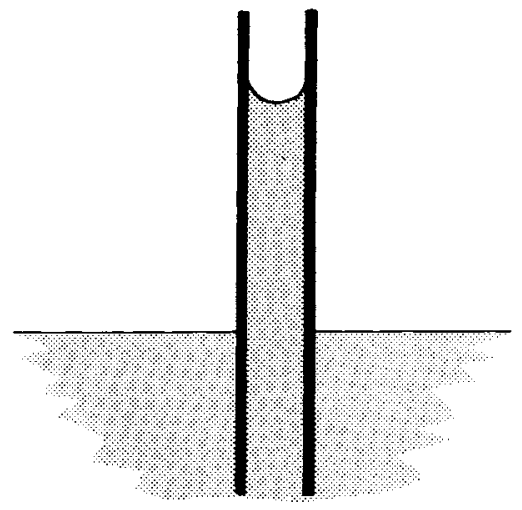

a

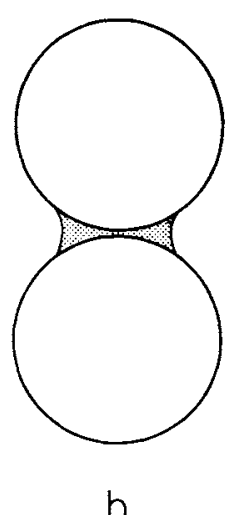

I socaps

FIg 3.- $a$, Ordinary connected capillary; $b$ and $c$, isocaps. 


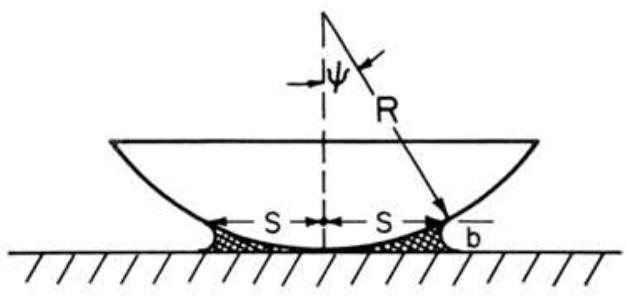

FIG 4.- Isocap formed between a spherical lens and a flat plate.

With low liquid volumes, the first term is minor and the final equation is identical to the Tabor equation ${ }^{2}$

$$
F=4 \pi R \gamma \cos \theta .
$$

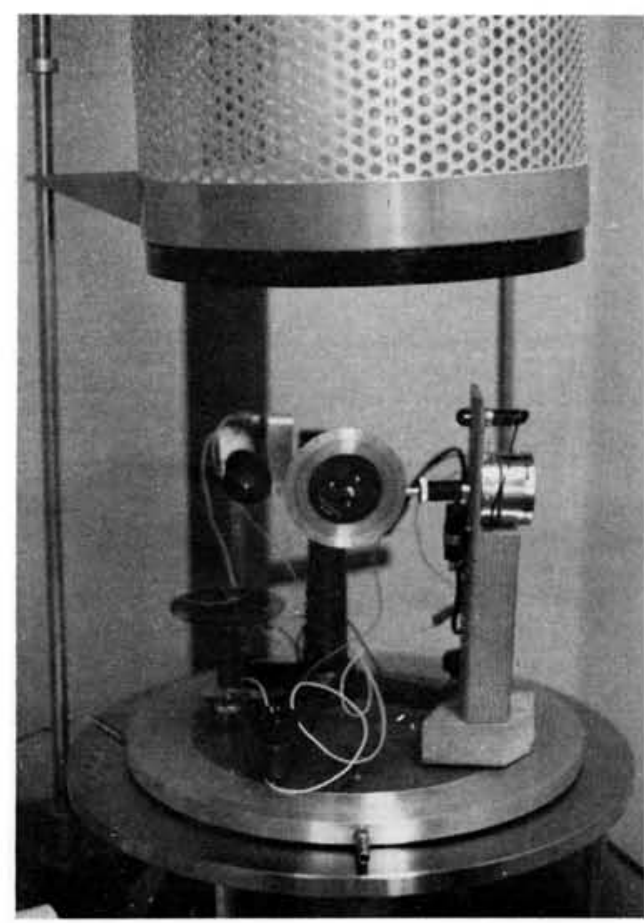

FIG 5.-Motor-driven surface tension balance and vacuum chamber.

\section{Materials and Methods}

EFFECT OF LIQUID VOLUME.-A surface tension torsion balance was modified to operate by means of a small $1 \mathrm{rpm}$ electric motor (Fig 5). An optically ground spherical lens with a radius of curvature of 17.9 $\mathrm{cm}$ and a flat glass plate were cleaned with chromate cleaning solution and rinsed in distilled water. The force of adhesion for different volumes of degassed distilled water was measured at atmospheric pressure ( 742 $\mathrm{mm} \mathrm{Hg}$ ).

EFFECT OF ATMOSPHERIC PRESSURE.After placing a selected volume, $0.141 \mathrm{ml}$, of water between the lens and plate, a bell jar was lowered over the apparatus and the pressure was reduced by means of a mechanical pump. A mercury manometer registered the pressure in the chamber. When the selected pressure was attained, the motorized surface tension balance was turned on and the tensile force necessary to rupture the bridge was measured.

ADHESION OF SALIVA.-The same equipment was used to measure the adhesive strength of human saliva that had a surface tension of 54 dynes $/ \mathrm{cm}$. A $0.141 \mathrm{ml}$ volume was tested. Several replications were made.

\section{Results}

EFFECT OF LIQUID VOLUME.-Table 1 gives the adhesion values with various water quantities. The calculated strength according to equation 10 was $15.8 \times 10^{3}$ dynes for $\psi=4^{\circ}$ and $\theta=10^{\circ}$. At volumes of about $0.141 \mathrm{ml}$, the adhesion was relatively constant.

EFFECT OF ATMOSPHERIC PRESSURE.Table 2 gives the data for adhesion under reduced pressure. These results indicate an insignificant effect on adhesive strength.

ADHESION OF SALIVA.-The average of six adhesive force measurements was $12.6 \times$ $10^{3}$ dynes. The predicted result calculated from equation 10 was $12.5 \times 10^{3}$ dynes. The adhesion of saliva does not show any

TABLE 1

Adhesion Values with Various Quantities of Water

\begin{tabular}{|c|c|c|c|c|c|c|c|c|}
\hline Volume (ml) & 1.13 & 0.846 & 0.564 & 0.423 & 0.282 & 0.141 & 0.100 & 0.05 \\
\hline $\begin{array}{c}\text { Force (Dynes } \\
\left.\times 10^{-3}\right)\end{array}$ & 13.8 & 13.9 & 14.4 & 14.9 & 15.3 & 15.9 & 15.8 & 15.6 \\
\hline
\end{tabular}


anomalous behavior in regard to adhesion and its behavior can be predicted by equation 10 .

\section{Discussion}

The results help to clarify the behavior of liquids in isolated capillaries. Although the tested equation may be derived on the basis of reduced liquid pressure beneath a concave meniscus, the experimental results indicate that atmospheric pressure plays an insignificant role. The derivation presented indicates that isolated liquid-solid interaction results in an increase in liquid surface tension and this is responsible for the increase in adhesion. This approach is supported by the low vapor pressures found with liquids in isolated capillaries. ${ }^{6}$ Also, recent data indicate that a redistribution of vacancies takes place in isolated capillaries. ${ }^{7}$ These light-scattering experiments showed a desorption of vacancies at the solid-liquid interfaces.

Saliva is present in isolated capillaries between and around teeth and under restorations. The change in surface tension of

TABLE 2

Adhesion Values with Reduced Pressure

\begin{tabular}{lccccccc}
\hline $\begin{array}{l}\text { Pressure } \\
(\mathrm{mm} \mathrm{Hg})\end{array}$ & 742 & 600 & 500 & 400 & 300 & 200 & 100 \\
$\begin{array}{l}\text { Force (Dynes } \\
\left.\times 10^{-3}\right)\end{array}$ & 15.8 & 15.9 & 15.9 & 15.9 & 15.9 & 15.9 & 15.9 \\
\hline Note: Water, 24 C; & $0.141 \mathrm{ml}$.
\end{tabular}

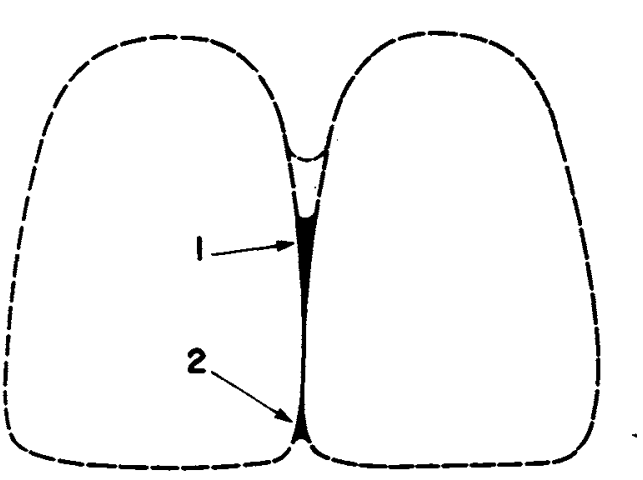

(a)

$$
\gamma^{*}-\gamma=\frac{2 \psi \gamma}{\pi(1-\cos \psi)}
$$

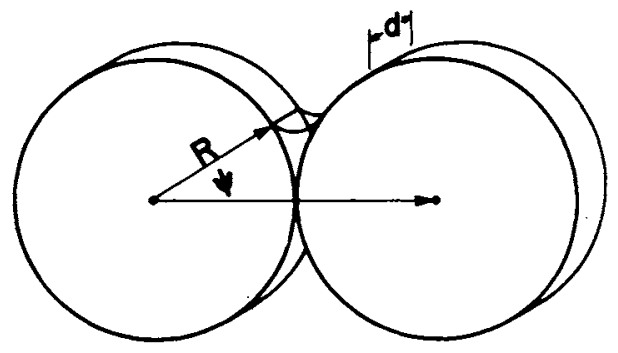

(b)

FIG 6.- Isocap formed between central incisors $(a)$ and geometric model consisting of two disks $(b)$.

$$
\gamma^{*}-\gamma=\frac{\gamma \ln \psi}{(1-\cos \psi)}
$$

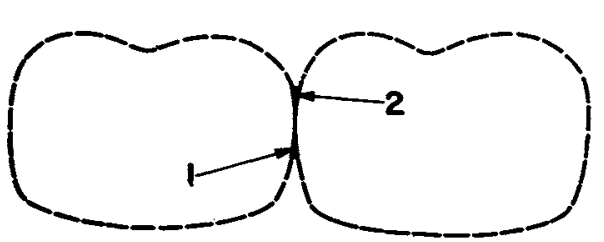

(a)

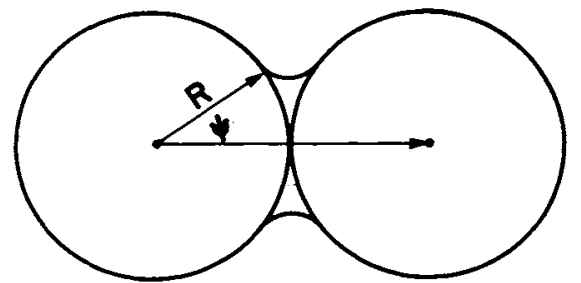

(b)

FIG 7.--Isocap formed between contacting molars (a) and geometric model of two contacting spheres $(b)$. 


$$
\gamma^{*}-\gamma=\frac{2 \psi \gamma}{\pi(1-\cos \psi)}
$$

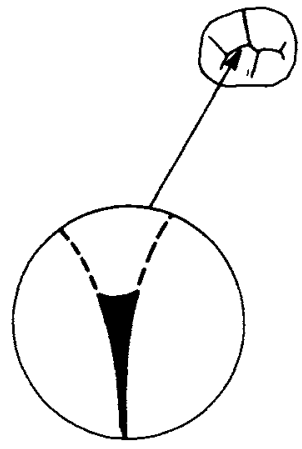

(a)

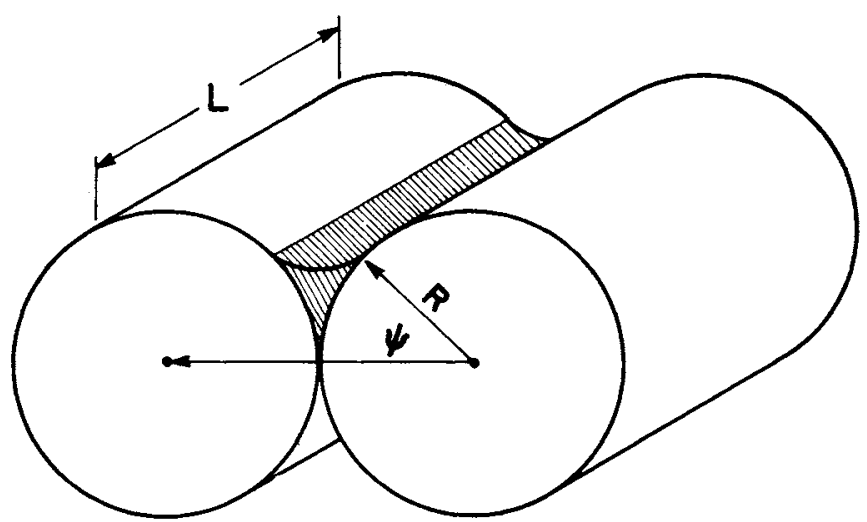

(b)

FIG 8.-Fissure isocap (a) and model consisting of two cylinders in contact $(b)$.

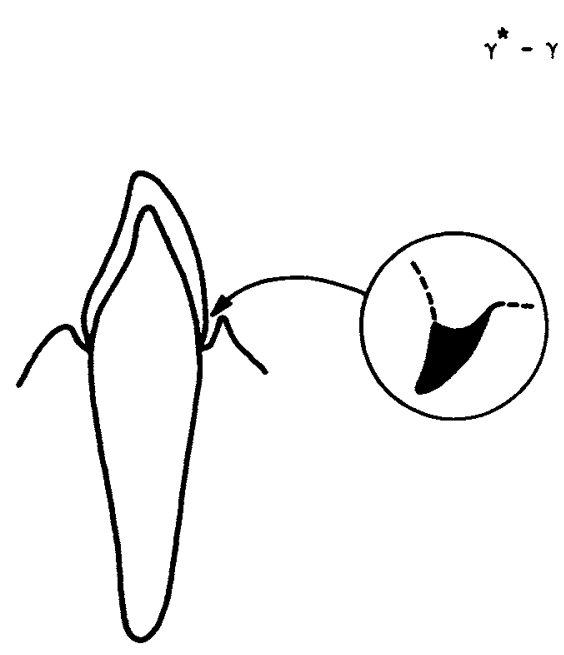

(a)

FIG 9.-Gingival isocap $(a)$ and model consisting of partially eclipsed spheres rotated about an axis $(b)$. 
saliva between adjacent teeth may be calculated by use of equation 2. Simplified geometric models and the expressions for the increase in surface tension are illustrated in Figures 6 and 7. Other isolated capillary systems form in occlusal fissures and gingival crevices, as indicated in Figures 8 and 9. The greatest effects may be expected when the amount of saliva is small. In a situation involving contacting teeth, the greatest surface tension increase occurs in embrasure regions that are highly curved. For example, the surface tension change would be higher in the gingival embrasure regions of maxillary incisors than in the regions of straighter mandibular incisors. A partially

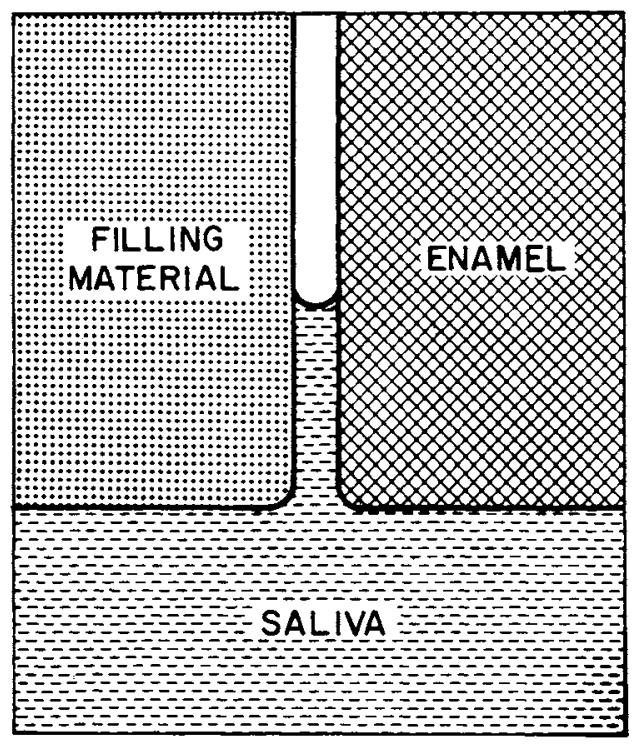

FIG 10.-Capillary penetration around filling. eclipsed sphere model is used for liquid isolated in gingival sulci.

Capillary penetration around filling materials is illustrated in Figure 10. The penetration pressure is governed by equation 1 . Decreasing the size of the crevice by improving adaptation will increase capillary pressure. Wettability offers a more feasible method of reducing marginal leakage. ${ }^{8}$

In the case of denture retention, the thin isolated layer of saliva between the denture and the mucosa (Fig 11) undergoes a change in properties. A decrease in pressure to less than atmospheric pressure is not necessary to explain this adhesion; the thinner the layer of liquid, the tighter the peripheral seal (this creates the isolated capillary) and the greater the wettability, the greater the adhesion. This component of denture retention, therefore, is a result of a strengthening of saliva caused by an increased intermolecular attraction at the liquid-vapor interface.

\section{Conclusions}

Capillary penetration of liquids into narrow crevices depends on the wetting of the solid by the liquid, surface tension of the liquid, and inversely, on the size of the opening. The properties of capillary liquid change when the liquid is trapped in isolated contact with a solid. The surface tension increases to a value $\gamma^{*}$. As a result, the strength of the liquid becomes much greater and the vapor pressure decreases. The strength of liquid bridges between solids may be derived on the basis of this surface tension increase, rather than on the basis of a negative pressure within the liquid film. Human saliva was found to have the tensile

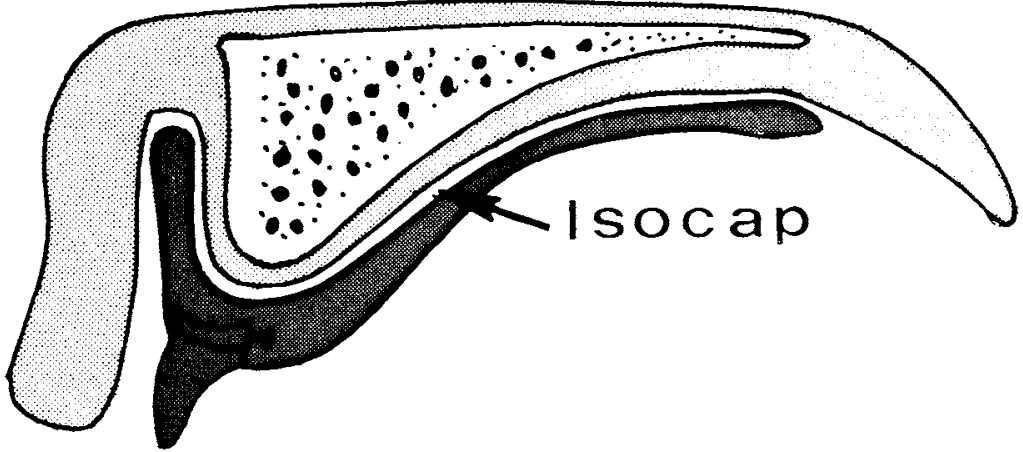

FIg 11.-Isocap space between denture and mucosa. 
strength predicted when derived on this basis.

Isolated capillaries are found in vivo around and between teeth and under dental restorations. Determination of the increase in liquid-surface tension in isolated capillaries at sites of caries susceptibility can be done mathematically. The penetration of saliva around dentures and marginal leakage result from the same phenomena. Pit and fissure sealants also enter crevices by capillary action.

Acknowledgement is due to $M$. Ash for the use of high volume vacuum apparatus.

\section{References}

1. O'Brien, W.J.; Craig, R.G.; and Peyton, F.A.: Capillary Penetration Between Dissimilar Solids, $J$ Colloid Interface Sci, 24:500$508,1968$.
2. Adamson, A.W.: Physical Chemistry of Surfaces, New York: Interscience Publishers, $1960, \mathrm{p} 9$.

3. O'Brien, W.J.: Surface Energy of Liquids Isolated in Narrow Capillaries, Surface Sci 19:387, 1970.

4. O'Brien, W.J.: Anomalous Behavior of Liquids in Isolated Contact with Solids, Surface Sci 25:298-304, 1971.

5. McFarlane, J.S., and Tabor, D.: Adhesion of Solids and the Effect of Surface Films, Proc $R$ Soc Lond A202:224-253, 1950.

6. Folman, M., and Shereshefsky: A Study of Vapor Pressure in Small Capillaries, $J$ Phys Chem 59:607, 1955.

7. Oliver, A.: Light Scattering by Liquids Isolated in Narrow Capillaries, Zeut Licht Elektronenoptik 32:218-236, 1970.

8. O'Brien, W.J.; Craig, R.G.; and Peyton, F.A.: Capillary Penetration Around a $\mathrm{Hy}-$ drophobic Filling Material, $J$ Prosthet Dent 19:399-405, 1968. 